\title{
Effects of Pulse-Synchronized Massage With Air Cuffs on Peripheral Blood Flow and Autonomic Nervous System
}

\author{
Osamu Tochikubo, MD; Senton Ri, MD; Naoki Kura, MD
}

\begin{abstract}
Background There are almost no reports on the physiological effects of the various electric massage devices available on the market. This report describes the effects on peripheral vascular circulation and autonomicnervous system activity of a pulse-synchronized air-massage (PS-AM) device in which cuffs apply air pressure cyclically to the lower limbs only during the cardiac diastolic phase.

Methods and Results The PS-AM device consists of a main unit with a pulse-wave detector that uses the pulse-wave to activate an air pump only during the cardiac diastolic phase. Pressure is applied sequentially from the feet to the thighs by 4 pairs of cuffs attached to the main unit. The study was carried out on 55 volunteers (19 males, 36 females). A laser Doppler flow-meter attached to the subjects' toes was used to measure blood flow $(\mathrm{BF})$, while ECG, blood pressure and cardiac autonomic nerve activity [parasympathetic nerve activity (highfrequency component (HF))] were measured by a multibiomedical recorder (TM2425). The measurements were made for a total of 45 min consisting of three 15-min periods: before massage, during PS-AM, and after massage. $\mathrm{BF}$ increased $139 \pm 33$ (SD) $\%$ in males and $154 \pm 44 \%$ in females during PS-AM, with a significant correlation being observed between BF and HF during PS-AM $(\mathrm{p}<0.01)$.
\end{abstract}

Conclusions PS-AM significantly increased peripheral vascular BF in the lower extremities, a change that may affect venous return and cardiac parasympathetic nerve activity. (Circ J 2006; 70: 1159-1163)

Key Words: Autonomic nervous system; Electric massage device; Peripheral vascular circulation; Plethysmogram

$\mathbf{N}$ umerous vibrator and air-cuff (pneumatic) massage devices for relieving fatigue and muscular stiffness are currently available on the market in Japan. However, as practically no research has been undertaken to investigate the effects of these devices on peripheral vascular circulation, their therapeutic mechanism remains unclear. In order to increase knowledge in this field, in 1994, in collaboration with a company named Advance (Tokyo, Japan), we developed a pulse-synchronized transpercutaneous electric muscle stimulator called a 'Venous Pump'! This device is a type of low-frequency electrical muscle-stimulator that measures the descending phase of the pulse-wave in the earlobe and provides low-frequency electrical stimuli to the muscle during the diastolic phase of the heart. The stimuli cause muscular contraction and promote the muscle-pump action ${ }^{2}$ in the peripheral circulation. At about the same time, working with Advance Co, we developed a second prototype of the system, a pulse-synchronized air-massage (PS-AM) device (Kaikan Slim) that provides pressure-stimuli to the lower extremities only during the cardiac diastolic phase. We anticipate that the PS-AM device accelerates venous return of blood in the lower extremities and changes parasympathetic system activity. The present study was designed to examine the

(Received December 26, 2005; revised manuscript received June 20, 2006; accepted July 7, 2006)

Department of Public Health, Yokohama City University School of Medicine, Yokohama, Japan

Mailing address: Osamu Tochikubo, MD, Department of Public Health, Yokohama City University School of Medicine, 3-9 Fukuura, Kanazawa-ku, Yokohama 236-0004, Japan. E-mail: tocchi@med. yokohama-cu.ac.jp physiological effects of PS-AM on peripheral circulation and autonomic nervous system activity.

\section{Methods}

The main unit of the PS-AM device consists of a photopulse sensor for the earlobe or the tip of a digit and an air pump that analyzes the pulse signal and inflates the cuffs round the lower limbs at the time of the cardiac diastolic phase (Fig 1). In addition, 4 hoses and 4 cuffs, one for each limb, transmit air pressure. Cuff pressure is released when blood is pumped from the heart to the peripheral arteries,

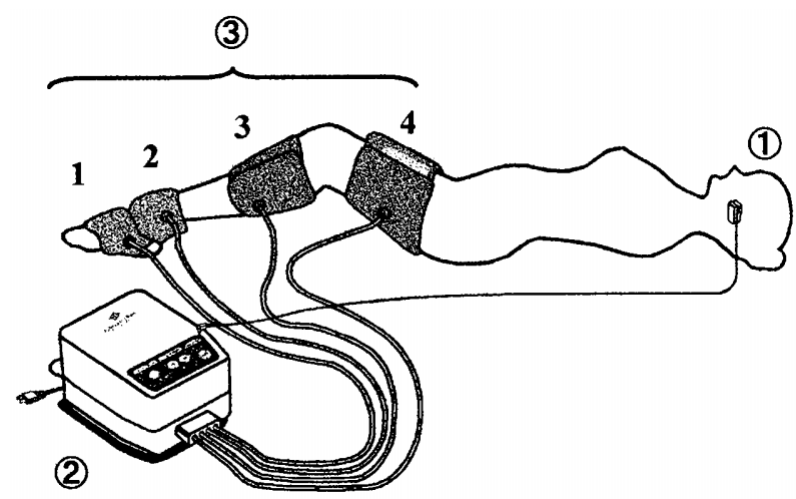

Fig 1. Pulse-synchronized air-massage device. (1)Pulse sensor, (2)air pump, (3)air-cuffs. The 4 pairs of air-cuffs are programmed to apply sequential pressure (beat-to-beat) continuously, starting from the feet to the thighs (from 1 to 4 ). 

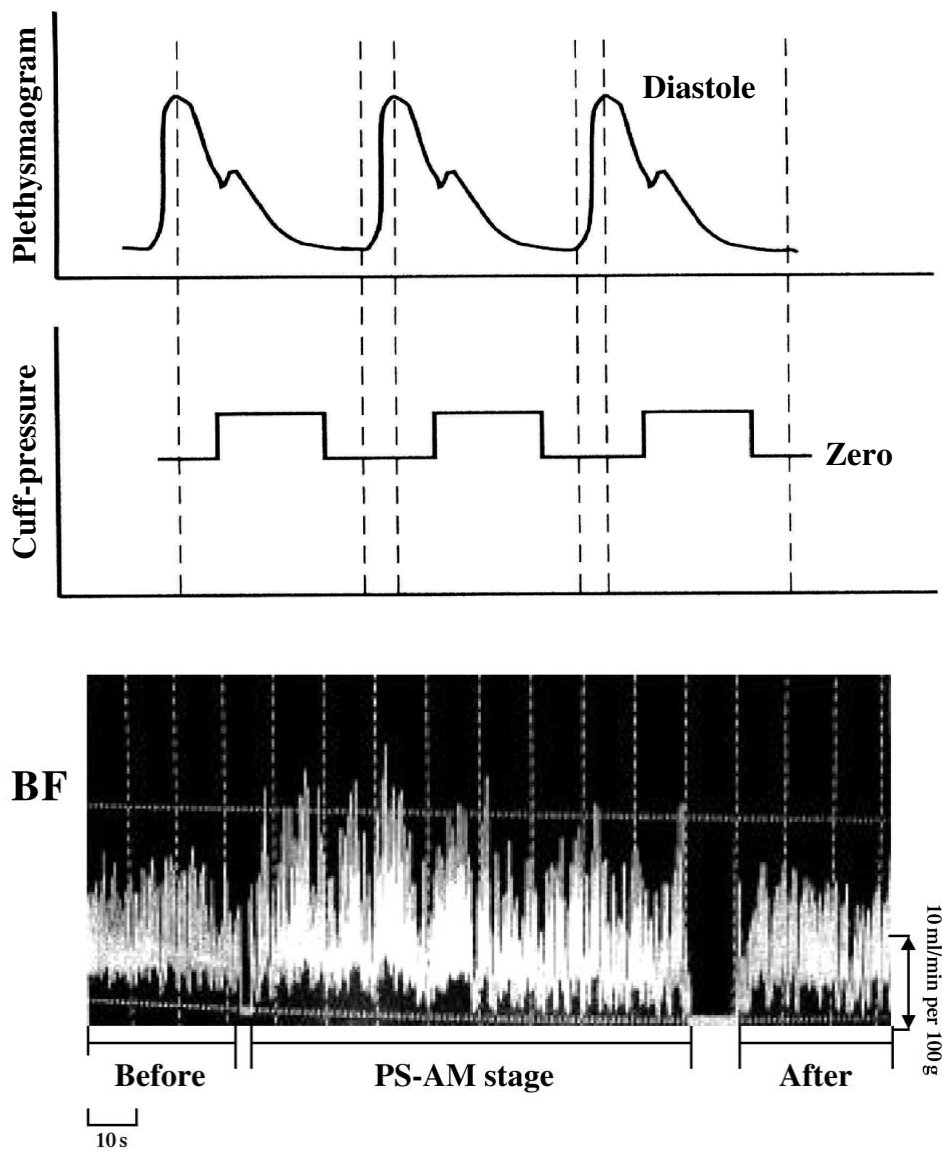

Fig 2. Increases in cuff-pressure are synchronized with a photo-plethysmogram. Cuff pressure is applied only during the descending segment of the pulse wave, which corresponds to the cardiac diastolic phase. No pressure is applied when arterial blood flows into the lower limbs during the cardiac systolic phase. corresponding to the systolic phase. The cuff inflates only during the diastolic phase when venous blood is returning to the heart, thereby reinforcing the muscle-pump action on the peripheral circulation of venous blood and lymph. Arterial pulse waves detected in the earlobe or digit tip determine the cardiac phases and activate the air pump to transmit pressure to the cuffs only during the diastolic phase (Fig 2). The diastolic phase is detected by the photo-pulse sensor that measures the peak and bottom of the arterial wave and triggers the air pump. Pressure in the cuffs can be regulated at 3 designated levels: low $(\approx 40 \mathrm{mmHg})$, normal $(60 \mathrm{mmHg})$ or high $(80 \mathrm{mmHg})$. This makes it possible to select a pressure that feels comfortable for the subject and only constricts the veins without affecting the arteries. The 4 cuffs applied to the legs (ie, a total of 8 cuffs) are programmed to apply sequential pressure for $15 \mathrm{~min}$ starting from the feet and continuing to the thighs in recurring cycles (beat-tobeat) (Fig 1). The subject lies prone on a bed and during the initial 15-min stage (pre-PS-AM stage) no cuff-pressure is applied. The PS-AM is then activated for $15 \mathrm{~min}$ (PS-AM stage), followed by a further 15-min during which no cuffpressure is applied (post-PS-AM stage).

Peripheral blood flow (BF), blood pressure (BP), and cardiac autonomic nerve activity were measured in this study by the following methods. Peripheral BF was measured continuously by a laser Doppler flow-meter (ALF21 model, Advance Co, Japan), with a BF laser sensor ${ }^{3,4}$ being applied to the underside of the third toes. The high-sensitivity photoelectric BF laser sensor uses an invisible semiconductor laser with a wavelength of $785 \mathrm{~nm}$. The flow-meter measurements expressed in units of $0-100 \mathrm{ml} / \mathrm{min}$ per $100 \mathrm{~g}$ were then entered into an Excel program on a personal com- puter and analyzed with WAAP-WIN software (ELMEC Co, Japan) that was capable of processing large volumes of data at high speed. Examples of BF curves derived from the Excel program are shown in Fig 3.

$\mathrm{BP}$ and cardiac autonomic-nervous system activity were measured from recordings of indirect BP and electrocardiograms (ECG) using a Multi-biomedical Recorder ${ }^{5}$ (TM2425, A\&D Co, Japan). BP was measured every 3 min during the PS-AM test. To obtain heart rate (HR) and cardiac autonomic nerve activity, the ECG-RR interval spectrum was analyzed. The low-frequency component (LF) was set between 0.4 and $0.15 \mathrm{~Hz}$, and the high-frequency component (HF) between 0.15 and $0.40 \mathrm{~Hz}$. The HF component was considered to represent parasympathetic-nervous activity and the LF/HF component to represent sympathetic-nervous activity. We have reported previously on the accuracy and methodology of these measurements?

All the subjects in the study were volunteers, and ranged

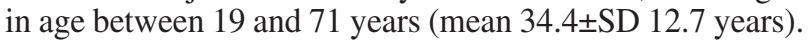
The 19 males and 36 females were studied separately, because mean BF levels differ between males and females. The study protocol was approved by the Ethics Committee of the School of Medicine of the Yokoyama City University, with informed consent being obtained from all participants prior to the study.

As a preliminary study, we selected 10 subjects ( 3 males, 7 females) from the original study group of 55 subjects and repeated the protocol using application of pressure at 1-s intervals without pulse synchronization. In the preliminary study, the BF in the toe increased marginally to $123 \pm 28 \%$ during the air massage stage. In contrast, cuff pressure with pulse-synchronization caused a significantly greater in- 
Table 1 Effects of PS-AM on BF and LF/HF

\begin{tabular}{|c|c|c|c|}
\hline & Before & $P S-A M$ & After \\
\hline \multicolumn{4}{|l|}{ Males $(n=19)$} \\
\hline$B F(\mathrm{ml} / \mathrm{min}$ per $100 \mathrm{~g})$ & $8.8 \pm 4.4$ & $12.0 \pm 6.4 * *$ & $11.9 \pm 6.0^{* *}$ \\
\hline $\operatorname{SBP}(\mathrm{mmHg})$ & $114.8 \pm 9.4$ & $114.2 \pm 11.0$ & $114.2 \pm 11.4$ \\
\hline$D B P(m m H g)$ & $71.2 \pm 12.1$ & $68.7 \pm 11.9$ & $68.8 \pm 12.1$ \\
\hline HR (beats/min) & $62.7 \pm 10.8$ & $59.3 \pm 10.0 * *$ & $59.9 \pm 9.6 * *$ \\
\hline$L F / H F$ (ratio) & $1.8 \pm 0.5$ & $1.3 \pm 0.7 * *$ & $1.7 \pm 0.6$ \\
\hline$H F\left(m s / H z^{1 / 2}\right)$ & $32.1 \pm 17.4$ & $39.0 \pm 19.7 * *$ & $35.4 \pm 17.4^{*}$ \\
\hline \multicolumn{4}{|l|}{ Females $(n=36)$} \\
\hline$B F(\mathrm{ml} / \mathrm{min}$ per $100 \mathrm{~g})$ & $7.4 \pm 6.2$ & $10.1 \pm 7.5^{* *}$ & $9.2 \pm 7.8 * *$ \\
\hline $\operatorname{SBP}(\mathrm{mmHg})$ & $106.2 \pm 8.4$ & $106.3 \pm 8.2$ & $106.6 \pm 7.9$ \\
\hline$D B P(m m H g)$ & $64.4 \pm 5.9$ & $63.7 \pm 7.3$ & $63.7 \pm 7.3$ \\
\hline HR (beats/min) & $66.8 \pm 7.9$ & $65.8 \pm 8.7 * *$ & $65.9 \pm 8.8 * *$ \\
\hline$L F / H F$ (ratio) & $1.3 \pm 0.5$ & $0.9 \pm 0.4 * *$ & $1.3 \pm 0.4$ \\
\hline$H F\left(m s / H z^{1 / 2}\right)$ & $29.3 \pm 11.6$ & $35.9 \pm 13.4 * *$ & $34.9 \pm 13.9 * *$ \\
\hline
\end{tabular}

Data are mean $\pm S D$.

$P S$-AM, pulse-synchronized air-massage; $B F$, blood flow; $L F / H F$, ratio of low frequency component of heart rate $(H R)$ variability to high frequency component of $H R$ variability; $S B P$, systolic blood pressure; DBP, diastolic blood pressure.

${ }^{*} p<0.05$, ** $p<0.01$ vs Before.
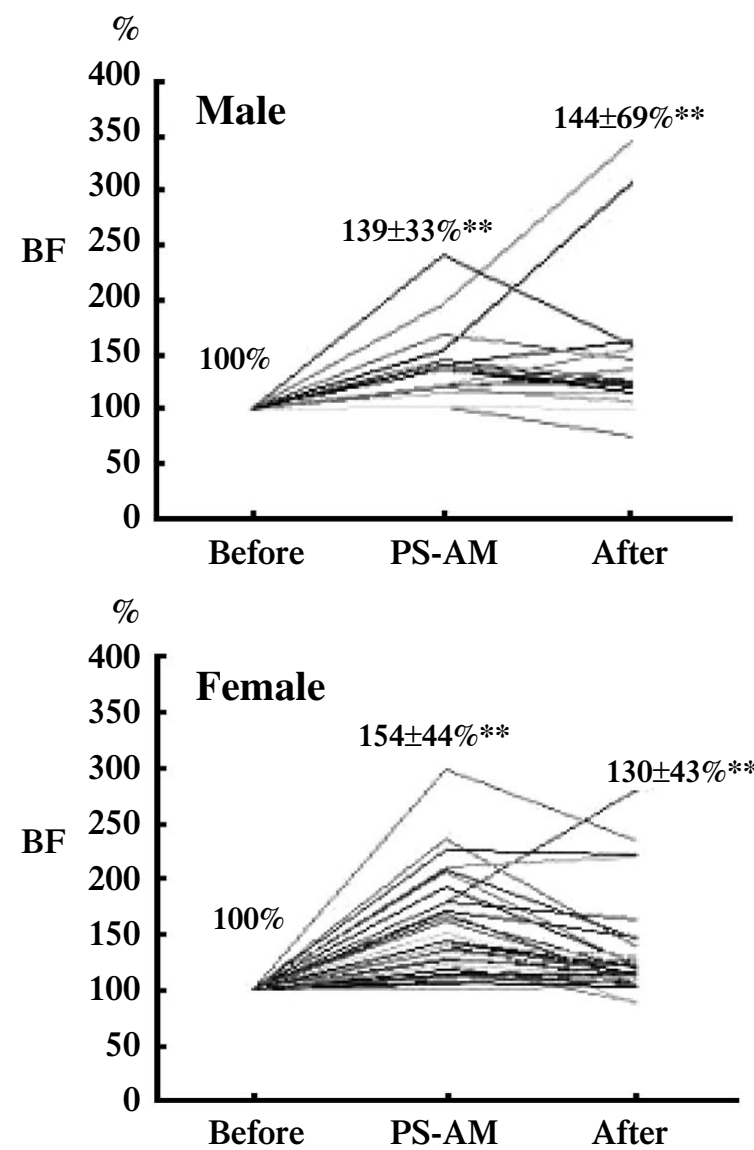

Fig 4. Percentage change in blood flow (BF) caused by pulsesynchronized air-massage (PS-AM). Before, pre-PS-AM stage; After, post-PS-AM stage. $* * \mathrm{p}<0.01$ vs Before.

crease in the BF in the toe $(165 \pm 42 \%, \mathrm{p}<0.01)$. The subjects also found the pulse-synchronization method more comfortable and for this reason it appeared pulse-synchronized air-cuff massage was the most effective method for increasing BF.

Standard statistical methods including paired 2-sample
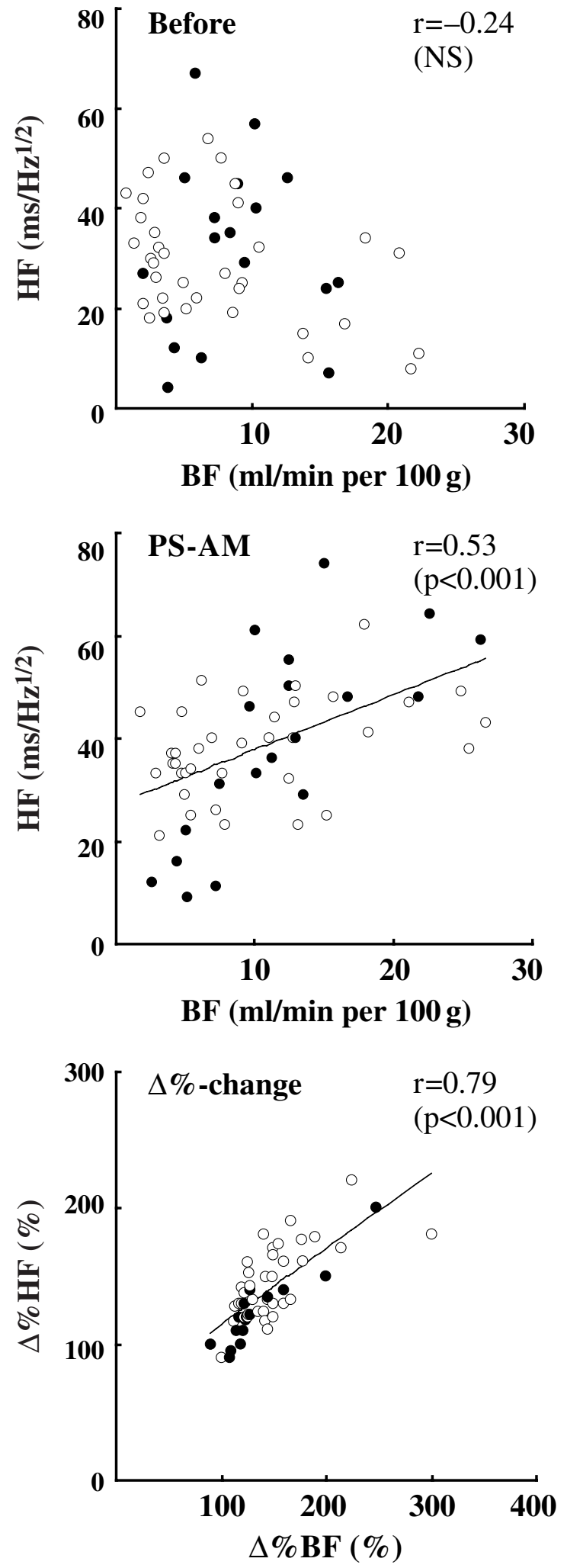

Fig 5. Relationship between mean blood flow (BF) and mean high frequency component (HF) of heart rate variability during pre-PSAM stage (Before) (Upper panel) and PS-AM stage (Middle panel), and between the percentage changes of $\mathrm{BF}[\Delta \% \mathrm{BF}=(\mathrm{BF}$ during PS$\mathrm{AM}-\mathrm{BF}$ during pre-PS-AM $/(\mathrm{BF}$ during pre-PS-AM $) \times 100 \%]$ and $\mathrm{HF}[\Delta \% \mathrm{HF}=(\mathrm{HF}$ during $\mathrm{PS}-\mathrm{AM}-\mathrm{BF}$ during pre-PS-AM $) / \mathrm{BF}$ during pre-PS-AM $\times 100 \%]$ (Lower panel). PS-AM, pulse-synchronized airmassage. Male; $\bigcirc$ Female. 
[A] Systolic phase

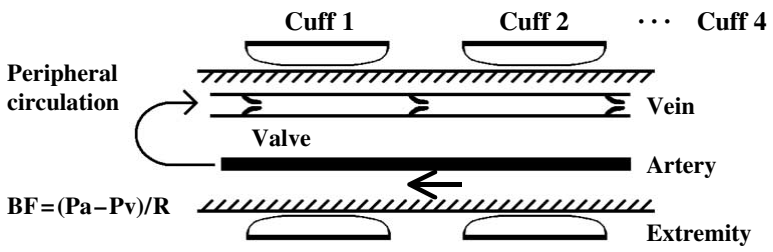

[B] Diastolic phase: compression of vein under cuff 1

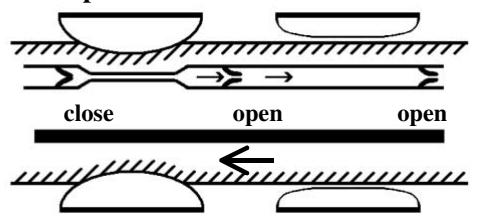

[C] Next diastolic phase: compression of vein under cuff 2

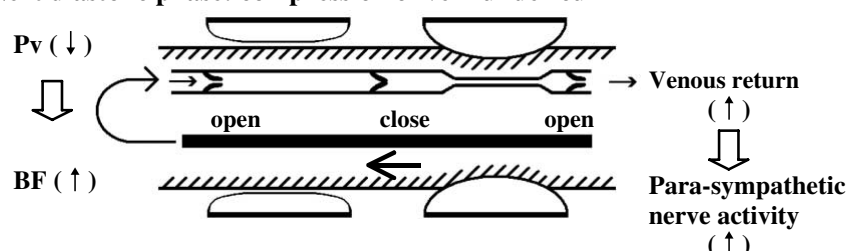

$(\uparrow)$
Fig 6. Hypothesis of the effects of the pulse-synchronized air-massage (PS-AM) device. BF, peripheral blood flow; $\mathrm{Pa}$, arterial blood pressure; $\mathrm{Pv}$, venous blood pressure; $\mathrm{R}$, total peripheral vascular resistance. The decrease in $\mathrm{Pv}$ caused by the PS-AM device may enhance BF and increase venous return, thereby stimulating parasympathetic nervous activity through a baroreflex function. t-tests, non-paired t-tests, F tests, ANOVA, and least-squares linear regression analysis were used in the analyses. The SPSS 11.0 for Windows (SPSS Japan Inc, Tokyo, Japan) program was used for these calculations. Values are expressed as mean $\pm \mathrm{SD}$, with values of $\mathrm{p}<0.05$ considered significant.

\section{Results}

Table 1 shows the mean BF values in the toe, systolic $\mathrm{BP}$, diastolic BP, HR, and cardiac autonomic nerve activity measured in the pre-massage resting stage, the PS-AM stage, and the post-massage resting stage in the male and female groups. Although no significant changes were observed in BP during the PS-AM stage in either group, BF increased significantly $(\mathrm{p}<0.01)$ during both the PS-AM and the post-massage stages compared to the pre-massage stage. Assuming the BF in the pre-massage stage was $100 \%$, the mean percentage change in BF during the PS-AM stage in males was $139 \pm 33 \%$ and in females $154 \pm 44 \%$ (Fig 4). These levels of percentage increases persisted during the 15-min post-massage stage (Table 1, Fig 4).

In contrast, HR decreased during PS-AM and tended to remain low throughout the post-massage stage. $\mathrm{LF} / \mathrm{HF}$ also decreased significantly $(\mathrm{p}<0.01)$ during the PS-AM stage (Table 1). It is thought that changes in HF exert a greater influence than changes in LF. We observed individual differences in the rate of BF change (Fig 4) and HF change. With the aim of determining the causes of these differences we measured the correlation coefficients (r) between the variables and showed a significant correlation between the mean $\mathrm{BF}$ and $\mathrm{HF}$ (males $\mathrm{r}=0.72$; females $\mathrm{r}=0.42$; all subjects $r=0.53, p<0.01$ ) and percentage changes in $\mathrm{BF}$ and $\mathrm{HF}$ (males $\mathrm{r}=0.83$; females $\mathrm{r}=0.68$; all subjects $\mathrm{r}=0.79, \mathrm{p}<0.01$ ) during PS-AM stage. However, there was no correlation between BF and HF during pre-PS-AM stage (Fig 5), which suggests that the rate of $\mathrm{BF}$ change contributes to cardiac autonomic nerve activity.

\section{Discussion}

Our hypotheses regarding the effects of PS-AM massage are described below (Fig 6). However, we consider there may be other unknown mechanisms that also have an influence on the changes we observed. Peripheral BF is expressed by the classical Poiseuille-Hagen equation: $\mathrm{BF}=\Pi(\mathrm{Pa}-\mathrm{Pv}) \mathrm{r}^{4} / 8 \mathrm{~L} \mu=(\mathrm{Pa}-\mathrm{Pv}) / \mathrm{R}\left(\mathrm{R}=8 \mathrm{~L} \mu \hbar \mathrm{r}^{4}\right)$, where $\mathrm{Pa}=$ arterial pressure, $\mathrm{Pv}=$ venous or capillary pressure, $\mathrm{r}=$ diameter of artery, $\mathrm{L}=$ length of artery, and $\mu=$ viscosity of blood (Fig 6A). Cuff 1-pressure $(40 \mathrm{mmHg})$ of the PS-AM device (Fig 6B) is applied to the lower part of the extremities only during the cardiac diastolic phase, with no pressure being applied when arterial BFs during the systolic phase. This intensifies the venous return by forcing blood through the counter current venous valve towards the central parts of the vein (under cuff 2), without disturbing arterial BF (Fig 6B). The vein under cuff 1 collapses, resulting in a decrease in venous pressure $(\mathrm{Pv})$ during the systolic phase, leading to an increase in $(\mathrm{Pa}-\mathrm{Pv})$. At the next beat, cuff 2-pressure is also applied only during the diastolic phase, forcing the blood towards the central parts of the vein (Fig 6C). These beat-to-beat actions are then applied sequentially to cuffs 3 and 4 . These mechanisms may enhance peripheral $\mathrm{BF}$ by decreasing $\mathrm{Pv}$, and also stimulate parasympathetic nervous system activity by increasing venous return as a consequence of baroreflex control.

The BF-promoting effects of the PS-AM device varied greatly between individuals (Fig 4). For example, in 4 males and 3 females, the BF was greater during the post-massage stage than in the PS-AM stage. These subjects experienced a sensation of warming in the lower limbs during the postmassage stage, but the reason for the increase of BF during the post-PS-AM stage in these subjects is unclear.

The ALF21 flow-meter used in this study uses a laser with a frequency of $785 \mathrm{~nm}$ that measures changes in BF in fine arteries near the skin surface. Vasomotion of 14 cycles/min 6,7 and the activity of sympathetic nerves in the 
skin have a marked influence on these blood vessels, which are known to constrict readily as a consequence of emotional stress? For this reason, we also measured LF/HF and $\mathrm{HF}$ as indices of cardiac sympathetic nerve activity at the same time as BF. HR variability and power spectral analysis were used as indices of sympathovagal balance in the heart9,10 Although LF/HF is not always proportional to skin and muscle sympathetic nerve activity and the reaction to stress ${ }^{11}$ we performed these measurements in order to investigate the relationship between $\mathrm{BF}$ and LF/HF. During the PS-AM operational stage, LF/HF decreased significantly, whereas HF increased.

Subjects with a high $\mathrm{HF}$, an index of parasympathetic nerve activity, also had high BF during the PS-AM stage (Fig 5). Many subjects reported feeling sleepy during PS-AM massage, possibly as a consequence of the rise in HF stimulating parasympathetic nerves. This indicates that when venous blood is transported towards the heart from lower-limb peripheral vessels, the Frank-Starling mechanism may come into effect. As stroke volume (SV) increases, although the change is a passive one, the baroreflex-control mechanism is activated. It is possible that parasympathetic nerve function is stimulated in order to lower HR and maintain a steady BP. The Frank formula ${ }^{12}$ is as follows: $\mathrm{BP}=$ cardiac output $\times \mathrm{TPR}=\mathrm{SV} \times \mathrm{HR} \times \mathrm{TPR}$, where $\mathrm{TPR}=$ total peripheral vascular resistance. We consider it is possible that HR and TPR decrease as a baroreflex action ${ }^{13}$ in order to maintain a steady BP during the PS-AM stage. This possibility is illustrated in Fig 5 in which the greater the increase in $\mathrm{BF}$ (with increasing venous return), the more $\mathrm{HF}$ (ie, parasympathetic nerve activity) is stimulated.

We found the PS-AM device was more effective in some cases than in others (Fig 4), possibly as a consequence of psychological tension causing variable degrees of autonomic nerve activity, which in turn would influence the peripheral circulation. The majority of subjects felt comfortable and sleepy when the PS-AM was running, suggesting that this method promotes relaxation.

This study measured BF only in fine arteries near the dermal surface layer. To verify the effectiveness of the method, further studies are required to measure factors such as venous BF, cardiac output, and TPR.

Air massage (pneumatic massage) is already used postsurgically to prevent venous stasis, phlebothrombosis of the lower limbs, and lymphedema of the limbs!4-17 As the PS-AM has a demonstrable effects it may be useful for improving peripheral circulation.

\section{Acknowledgment}

The authors wish to thank Advance Co, Tokyo, Japan, for creating and providing the pulse-synchronized air-massage device (Kaikan Slim).

\section{References}

1. Tochikubo O, Miyajima E, Okabe K, Imai K, Ishii M. Improvement of multiple coronary risk factors in obese hypertensives by reduction of intra-abdominal visceral fat. Jpn Heart J 1994; 35: 715-725.

2. Doran FSA, Drury M, Sivyer A. A simple way to combat the venous stasis which occurs in the lower limbs during surgical operations. $\mathrm{Br}$ J Surg 1964; 51: 486-492.

3. Kasima S, Nishihara M, Takemoto Y, Toshihiko O. Relationship between the integrated intensity of the power spectrum of light and tissue blood volume by the dynamic light scattering method. Jpn J Appl Phys 1990; 29: 1862-1865.

4. Kasima S, Soiida A, Takeuchi H, Toshihiko O. Study of measuring the velocity of erythrocytes in tissue by the dynamic light scattering method. Jpn J Appl Phys 1993; 32: 290-295.

5. Tochikubo O, Ikeda A, Miyajima E, Ishii M. Effects of insufficient sleep on blood pressure monitored by a new multibiomedical recorder. Hypertension 1996; 27: 1318-1324.

6. Asano M, Branemark PI. Microphotoelectric plethysmography using a titanium chamber in man. Adv Microcirc 1972; 4: 131-160.

7. Fagrell B, Fronek A, Intaglietta M. A microscope-television system for studying flow velocity in human skin capillaries. Am J Physiol 1977; 233: H318-H321.

8. Ludbrook J, Vincent AH. The effect of mental arithmetic on hand blood flow. Aust J Exp Biol Med Sci 1974; 52: 679-686.

9. Yanagi S, Yoshinaga M, Horigome H, Tanaka Y, Fusazaki N, Matsuoka Y, et al. Heart rate variability and ambulatory blood pressure monitoring in young patients with hypertrophic cardiomegaly. Circ J 2004; 68: 757-762.

10. Hadase M, Azuma A, Zen K, Asada S, Kawasaki T, Kamitani T, et al. Very low frequency power of heart rate variability is a powerful predictor of clinical prognosis in patients with cognitive heart failure. Circ J 2004; 68: 343-347.

11. Grassi G, Turri C, Vailati S, Dell'oro R, Mancia G. Muscle and skin sympathetic nerve traffic during the "white-coat" effect. Circulation 1999; 100: $222-225$.

12. Frank O. Die ground Form des arteriellen Pulses. Zschr F Biol 1899; 37: 483-526.

13. Tochikubo O, Kawano Y, Miyajima E, Nagura T, Ishii M. Circadian variation of hemodynamics and baroreflex functions in patients with essential hypertension. Hypertens Res 1997; 20: 157-166.

14. Yamazaki Z, Idezuki Y, Nemoto T, Togawa T. Clinical experiences using pneumatic massage therapy for edematous limbs over the last 10 years. Angiology 1988; 39: 154-163.

15. Felty CL, Rooke TW. Compression therapy for chronic venous insufficiency. Semim Vasc Surg 2005; 18: 36-40.

16. Calnan JS, Pffug JJ, Mills CJ. Pneumatic intermittent compression legging simulating calf muscle pump. Lancet 1970; 2: 502-503.

17. Mittelman JS, Edwards WS, McDonald JB. Effectiveness of leg compression in preventing venous stasis. Am J Surg 1982; 144: 611613. 